\title{
A Language for Array and Vector Processors
}

\author{
R. H. PERROTT \\ NASA/Ames Research Center
}

The scientific community has consistently demanded from computing machines an increase in the number of instructions executed per second. The latest increase has been achieved by duplication of arithmetic units for an array processor and the pipelining of functional units for vector processors. The high level programming languages for such machines have not benefited from the advances which have been made in programming language design and implementation techniques.

A high level language is described in this paper which is appropriate for both array and vector processors and is defined without reference to the hardware of either type of machine. The syntax enables the parallel nature of a problem to be expressed in a form which can be readily exploited by these machines. This is achieved by using the data declarations to indicate the maximum extent of parallel processing and then to manipulate this, or a lesser extent, in the course of program execution. It was found to be possible to modify many of the structured programming and data structuring concepts for this type of parallel environment and to maintain the benefits of compile time and run time checking. Several special constructs and operators are also defined.

The language offers to the large scale scientific computing community many of the advances which have been made in software engineering techniques while it exploits the architectural advances which have been made.

Key Words and Phrases: array processing, vector processing, parallel data structures, parallel control structures

CR Categories: 4.20

\section{INTRODUCTION}

During the last two decades, the design and development of several generations of computers have given rise to increased processing speeds; the more recent advances in the number of operations performed per second have been obtained by a revolution in computer architecture rather than by component technology. Examples of this revolution are the duplication of arithmetic units for array processors such as the Illiac IV [1] and the Phoenix [2], and the pipelining of functional units for vector processors such as the Star-100 [3] and the Cray-1 [10].

Permission to copy without fee all or part of this material is granted provided that the copies are not made or distributed for direct commercial advantage, the ACM copyright notice and the title of the publication and its date appear, and notice is given that copying is by permission of the Association for Computing Machinery. To copy otherwise, or to republish, requires a fee and/or specific permission.

This work was done while the author was on leave of absence from The Queen's University of Belfast and was with the Institute for Advanced Computation, NASA/Ames Research Center, Moffett Field, CA 94035 .

Author's address: Department of Computer Science, The Queen's University, Belfast BT7 1NN, Northern Ireland.

(C) 1979 ACM 0164-0925/79/1000-0177 $\$ 00.75$

ACM Transactions on Programming Languages and Systems, Vol. 1, No. 2, October 1979, Pages 177-195. 
These types of computer are based on a form of parallel or lockstep processing which does not have the synchronization problems of a conventional multiprocessor system. These machines are widely used in large scale scientific computations, particularly for grid or mesh type problems where regularity of processing the data is the dominant problem characteristic; they form the baseline architecture for this paper. Hence asynchronous parallel configurations such as C.mmp [15] are excluded from consideration.

Unfortunately, there has not been a comparable investment of either research funds or effort into the development of programming languages or software production tools to utilize these technological and architectural advances. Most of the high level languages currently used to program these parallel computers are extensions of languages which were specifically designed, many years ago, for sequential machine architectures, e.g., extended Fortran for the Star-100 [11], CFT for the Cray-1 [10], and IVTRAN [8], CFD [12] (Fortran-like languages), and Glypnir [7] (an Algol-like language) for the Illiac IV. SL/1 [6] is one of the few languages that has tried to bring some of the benefits of structured programming to one of these machines, namely, the Star-100.

The gap between the hardware and software development for these machines has been apparent in many of the projects attempted, e.g., some conversions of important production codes to the Illiac IV were terminated due to software problems $[5,13]$. Also the size and the complexity of the projects that programmers are being asked to implement have increased with the available processing power and are now almost beyond the features and capabilities of the programming languages being used to tackle them.

More specifically, to construct and to increase the efficiency of a program, the user either has to be aware of the machine instruction set or of the method of detection of parallelism used by the compiler. In addition, the organization of the transfer of data to and from the backing store can require the use of low level primitives; the transfer can critically affect the performance of a program. Hence the challenge to the language designer is to devise a language which provides the programmer with sufficient tools to enable the construction of efficient algorithms and at the same time effectively utilize the hardware.

The language described in this paper is an attempt to redress the technology imbalance: to develop a high level language whose features exploit the advanced architecture of these parallel machines and incorporate the new software engineering approaches that are necessary in writing algorithms in this parallel environment. The language is called Actus.

The new language enables the specification of parallelism directly. The features are appropriate for both array and vector processors and they are defined without reference to the hardware of either type of machine; the algorithmic and data constructs are of sufficient generality and structure to make efficient use of the parallel computational resources. This could facilitate codes developed on one parallel architecture being moved to another parallel architecture without undue loss of efficiency. The language features of Actus are described using a notation similar to that of the language Pascal [14]. (A possible implementation is also suggested by using a Pascal P-compiler [9]; such an approach is currently being pursued at the Institute for Advanced Computation for one of the parallel computers, namely, the Illiac IV.) 
It is therefore hoped that this type of parallel computer can benefit from some of the advances of structured programming and software engineering which are, as yet, not widely disseminated throughout the scientific community and that this, in turn, will mean reduced software production costs, with the improved quality, reliability, and adaptability that have been realized for sequential machines.

\section{DESIGN APPROACH}

There have been two main approaches to the design of a high level language for a vector or an array processor which are reflected in the existing languages for these machines, namely, either

(i) the user writes a program in a conventional sequential programming language and the compiler tries to detect the inherent parallelism of the program, or

(ii) the parallel nature of the computer is readily apparent in the syntax of the language.

Examples of the first philosophy are IVTRAN for the Illiac IV and CFT for the Cray-1. Such languages try to extract the parallelism within sequential Fortran DO loops. The disadvantages of this approach are that the extraction of the parallelism is somewhat limited and inefficient in the code generated; and often the user has to restructure the program to benefit from the parallelism of the machine.

Examples of the second approach are CFD and Glypnir for the Illiac IV. CFD is an extension of Fortran which reflects in the syntax that the Illiac IV has 64 processors. The user then has to size the data structures of the problem to this natural length of the machine. This can add significantly to the complexity of a program, again causing the user to restructure the program.

Another example is Star Fortran. To access the parallel capability of the Star, the programmer must explicitly encode hardware instructions in separate subroutine calls (one call for each instruction). This effectively turns the extended Fortran into a higher level assembler language.

Another disadvantage in using these existing languages is that normally the two-level memory hierarchy is not a natural part of the language abstraction. For example, in the situation where the database under consideration is so large that it will not fit into the available main store, the user must, employing very basic facilities, organize the transfer of data between the extended memory and the fast processing store. Since the management of the memory hierarchy is often the critical factor in the overall performance of a program, the user must therefore code the most crucial aspect of the program in the most primitive syntax.

The approach adopted in the language reported here is that the language should enable the expression of parallelism in a manner which is suitable for the problem and can be easily exploited by these parallel machines. This will enable the compiler to generate efficient object code in a rapid and straightforward manner without resorting to a complex detection mechanism for the inherent parallelism. Another advantage is that programming with a parallel syntax produces algorithms of greater efficiency, i.e., a user does not have to size the data structures of the problem but can adopt a straightforward and natural notation to suit the problem. 
Thus the current programming situation for these parallel computers is such that the user is forced both to construct a solution to a problem using a language which does not provide the most appropriate abstraction mechanisms and to take account of the particular hardware characteristics of the machine.

\section{LANGUAGE CRITERIA}

The design of the language involved an extensive study of the problem areas in which such computers are used, consultations with programmers who had used such machines for many years, and a survey of the users of the Illiac IV over the years that it had been operational. The survey responders included users of both types of language as represented by the pair CFD/Glypnir and IVTRAN. The main points were

(i) parallel computations were difficult to express in or adapt to the syntax of the language if they were not based on a factor of 64 , i.e., the number of processing elements available,

(ii) manipulation of the status of processing elements was cumbersome; frequently data structures were increased in size to avoid status manipulation,

(iii) the lack of suitable control structures caused complexity of programming and at times required the introduction of machine code; machine code was also introduced to increase efficiency,

(iv) selecting an arbitrary group of array elements was difficult,

(v) manipulation of part words required machine code,

(vi) detailed knowledge of the layout of the data on the backing store and in the main store is required; also the facilities for specifying the transfer of data were primitive and required careful programming,

(vii) debugging and tracing facilities were lacking.

The purpose of the study, the consultations, and the survey were designed to determine the frequency of use of certain features and to identify what new features were required. In this way the relevant abstractions for large scale scientific problems could be formed along with their representation and rules for manipulation. This, in turn, led to the introduction of new data structures, language constructs, and operators. In effect, the new features were involved with manipulation of the extent of parallel processing, while the normal structured programming concepts were modified to enable representation and manipulation of the data in parallel.

The following criteria were adopted for the design of a language for the current parallel computers:

(i) the idiosyncrasies of the hardware should be hidden from the user as much as possible,

(ii) the user should be able to express the parallelism of the problem directly,

(iii) the user should be able to think in terms of a varying rather than a fixed extent of parallel processing,

(iv) control of the parallel processing should be possible both explicitly and through the data, as applicable,

(v) the user should be able to indicate the minimum working set size of the ACM Transactions on Programming Languages and Systems, Vol. 1, No. 2, October 1979. 
database (in those cases where the database is larger than the size of the fast memory).

The implementation of the language should provide compile and run time checks plus tracing facilities to assist with the debugging and testing of programs. Since lockstep parallel computers were developed as a means of performing the same operation on independent data sets in parallel, in Actus the data declarations indicate the maximum extent of parallelism. The extent of parallelism is defined for an array processor as the number of processors that can logically compute upon a particular data at the same time (this can be less than, equal to, or greater than the actual number of processors); for a vector processor it is the length of the data structure presented to the processor for computation.

Hence each data declaration has associated with it a maximum extent of parallelism; the language statements or constructs can then adjust this maximum (or a lesser) extent of parallelism in the course of program execution. In this way it is possible to express directly in the syntax the parallel nature of an algorithm which is appropriate for both vector and array processors.

The extent of parallelism can be regarded as representing a second programming dimension which can be adjusted in the course of program execution; the first dimension is that represented in a sequential programming language, namely, the successive execution of statements.

\section{DATA TYPES}

The examination of the problem area indicated that in addition to sequential data types a parallel data structure was required in which it was possible

(i) to associate parallel processing with any of the dimensions of the structure,

(ii) to enable accessing of a two- (or higher) dimensional structure other than by rows or columns, in parallel, and

(iii) to enable the combination of neighboring elements of a parallel structure.

To further facilitate the assignment and modification of the extent of parallelism, parallel constants and index sets, respectively, were introduced.

The language supports standard, enumerated, subrange types, and structured types such as the array and record (without the variant part) as defined in the language Pascal [4]. In addition, standard short integer and short real types requiring half the storage of their corresponding full word types were introduced (if supported by the hardware). All such variables are referred to as scalar variables.

The array declaration is used to indicate the maximum extent of parallelism for the data; in principle, the syntax can support any number of dimensions, but the examples are restricted to three, or fewer.

A scalar array is represented as

var scalara: array $[1 . . m, 1 . . n]$ of integer;

i.e., scalara contains $m \times n$ (predefined) integer numbers. The lower indices are restricted to 1 for convenience. The maximum extent of parallelism is introduced 
by replacing one (and only one) pair of sequential dots ".." by a parallel pair ":"

$$
\text { var para: array }[1: m, 1 \ldots n] \text { of real; }
$$

indicates an array para of $m \times n$ real numbers for which the maximum extent of parallelism is $m$. The array para can be manipulated $m$ elements at a time since it has been declared as a parallel variable with that extent of parallelism. The array is thus the main data structure to indicate variables which can be manipulated in parallel.

This means of representing the extent of parallel processing had a major influence on the design of the language. Previous to its discovery, special reserved words had been used to indicate parallel variables, for example, row-vector or column-vector prefixes. This, in turn, required the introduction of new control structures to facilitate the manipulation of parallel variables, for example, a where construct to select various elements of a row or column vector.

However, the rearrangement of one pair of dots in the data declaration considerably simplified and reduced the number of control structures. As can be seen in a later section, it is possible to use the same control structures for both scalar and parallel variables provided an extent of parallelism is associated with the parallel variables.

More specifically, in the declaration

var $a:$ array $[1: 4,1 . .5]$ of integer;

the parallel dots in the first index indicate that up to four elements of $a$ can be processed in parallel; the elements are selected one from each row and the second index selects the particular column (which can be the same or different for each row; see Section 4.2).

Thus $a[1: 4,2]$ is equivalent to referencing in parallel

$$
a[1,2] a[2,2] a[3,2] a[4,2],
$$

and $a[2: 3,1]$ is equivalent to referencing in parallel $a[2,1] a[3,1]$. Hence, the parallel dots indicate the index which is to be processed in parallel, that is, spread across the processors in an array processor or stored contiguously in a vector processor. The user can now indicate the index of an array with which the parallel processing is associated. Each time a parallel variable is used in a statement, it can have an extent of parallelism less than or equal to its maximum (or declared) extent of parallelism. In this way it is possible in the program text to indicate directly a parallel variable and to easily distinguish between parallel variables and scalar variables, e.g., other arrays or variables.

Binary operators can be applied to parallel variables if the extent of parallelism is the same for both operands or if one of the operands is a scalar variable or constant.

During the design of the language the possibility of having two or more pairs of parallel dots in a declaration was considered, e.g.,

$$
p p: \text { array }[1: m, 1: n] \text { of real; }
$$

This would indicate that the array $p p$ was intended to be manipulated in parallel by both its first and its second indices, i.e., $m$ elements at a time and also $n$ 
elements at a time either separately or concurrently in the course of program execution. On an array processor, to increase its efficiency, this would require that the data either be transposed during the calculation or skewed when being arranged in the stores. It was decided to restrict the parallelism to one dimension until more experience was gained in the use and the implementation of the language.

Thus the data declarations of Actus allow a flexible choice of index limits for each dimension of an array and enable the user to decide which dimension can be accessed in parallel. Such flexibility is absent in other languages such as CFD and Glypnir, where only the first dimension can be manipulated in parallel and then only with a fixed extent of parallelism, i.e., 64 .

\subsection{Parallel Constants}

In addition to the definition of scalar constants, identifiers can be defined to represent a sequence of integer numbers; they are used to assign values to parallel variables with an extent of parallelism equal to the number of values. The form of a parallel constant is

$$
\text { const } \text { identifier }=\text { start: }(\text { increment }) \text { finish; }
$$

where the values of start, increment, and finish must be integers and the sequence is

$$
\text { start, start }+ \text { increment, start }+2 * \text { increment }, \ldots, \text { finish. }
$$

If the increment is unity, it may be omitted, e.g.,

$$
\text { const } n=50 ; s e q=1: n \text {; oddseq }=1:(2) 31 ;
$$

Parallel constants can be used to assign values to parallel variables, for example, seq with an extent of parallelism 50 and oddseq with an extent 16.

\subsection{Independent Indexing}

One-dimensional parallel integer arrays can be used for independent indexing in a two- (or higher) dimensional parallel variable, thus enabling other parts of an array other than a row or column to be accessed. This is achieved by assigning appropriate values to a one-dimensional array and then using it as an index of a parallel variable. The extent of parallelism for both the indexing and the indexed array must be the same. For example,

$$
\begin{aligned}
& \text { const diagonal = } 1: 100 ; \\
& \text { var diag: array }[1: 100] \text { of integer; } \\
& \text { para: array }[1: 100,1 \ldots 100] \text { of real; }
\end{aligned}
$$

then $\operatorname{diag}[1: 100]:=$ diagonal assigns element $i$ of the array diag the value $i$, and para [1:100, diag [1:100]] accesses the diagonal elements of the array para. Hence any selection of elements of an array can be accessed as determined by the indexing array. Such a mechanism is necessary when it is required to access the off-diagonal elements of an array, for example.

\subsection{Index Sets}

The extent of parallelism can be changed each time a parallel variable is referenced; this is done by explicitly changing the extent of parallelism or by 
using an "index set" which identifies the data elements that are to be altered. The members of an index set are (ordered) integer values, each of which identifies a particular element of a data type that can be accessed in parallel.

An index set is defined with the data declarations and takes the form

$$
\text { index } \operatorname{ind} x=i: j \text {; }
$$

where $i$ and $j$ are constant integer values such that $i<=j$. The elements $i$ to $j$ inclusive will be accessed whenever the index identifier ind $x$ is used as a parallel array index. For example, with the declarations

$$
\begin{aligned}
& \text { var para: array }[1: m, 1 \ldots n] \text { of real; } \\
& \text { index interior }=2: m-1 ;
\end{aligned}
$$

interior can be used as the first index of the array para to access column elements other than the boundary elements.

Specific elements can be excluded from an index set definition by using a comma to indicate a break in the range. Also, if there is regularity in the required parallel index, this can be defined in an index set by inserting the increment in parentheses between the two terminal values. For example,

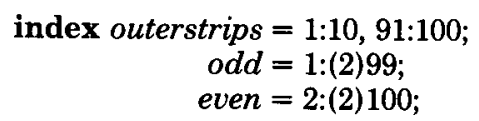

would enable the manipulation of various parts of an array with an extent of parallelism 1 to 100 (at least).

The advantages of using index sets are that

(i) statements become more readable since they use the identifier name, and

(ii) the extent of parallelism involved can be evaluated before the statement is encountered (and thus may permit greater efficiency in execution).

Hence an index set indicates an extent of parallelism which is less than or equal to the declared extent of parallelism of the variable being accessed.

Index set identifiers cannot be redefined, but they can be operated upon by union $(+)$, intersection $(*)$, and difference $(-)$ in order to facilitate computation on various parts of a parallel structure. The complement $(-)$ gives the other members of the declared extent of parallelism.

\subsection{Alignment of Data}

In order to enable the movement of data between elements of the same or different parallel variables, two primitive data alignment operators are included in the language. These are

(i) the shift operator which causes movement of the data within the range of the declared extent of parallelism, and

(ii) the rotate operator which causes the data to be shifted circularly with respect to the extent of parallelism.

The general form of the parallel index using these binary operators is

eop alignment operator distance

ACM Transactions on Programming Languages and Systems, Vol. 1, No. 2, October 1979. 
where eop is either an explicit definition of the extent of parallelism or an index set, and distance is a positive or negative integer expression.

The indices of the data to be moved (the source indices) are eop + distance and the destination indices are eop. Hence if distance is positive, it causes data to be moved from right to left, while if it is negative, it causes data to be moved from left to right. For example, using

var parb: array [1:100] of integer;

index first $50=1: 50$;

then $\operatorname{parb}[$ first50] + parb [ first50 shift 1] causes elements 1 to 50 of parb to be added to elements 2 to 51 , respectively, in parallel, while

$$
\text { parb }[\text { first } 50]+\text { parb }[\text { first } 50 \text { rotate }-1]
$$

causes element 1 to be added to 50 , and elements 2 to 50 to be added to 1 to 49 , respectively, in parallel.

To avoid any ambiguities in the application of alignment operators, all the indices involved in a statement are evaluated and the data values determined before the statement is executed. In this way alignment operations which indicate or try to access nonexistent (out of range) elements can be trapped.

In the other parallel languages there is no direct equivalent of index sets or the shift operator; the rotate operator is the main intercommunication mechanism. There are no formal parallel constants either, but a DATA statement can be used in CFD for assignment to particular variables; a reserved constant PEN which contains the values 0 to 63 can be used in Glypnir. A restricted form of independent indexing is available in both languages, i.e., along a nonparallel dimension. Once again the parallelism is in terms of 64 simultaneous operations.

\section{STATEMENTS}

In conjunction with the choice of data structures (and operators), the rules for their manipulation in the course of program execution were also formed. It was found that to construct algorithms for these parallel machines, many of the capabilities which are required in a sequential environment such as assignment, selection, iteration, procedure, and function abstractions are necessary. The essential difference is that in the new environment such manipulations must be performed in parallel. After many attempts, this was found to be possible by taking the syntax and semantics for sequential constructs and expanding them to include the extent of parallelism. This enables the manipulation of the extent of parallelism by means of the data; additional features were required to enable direct manipulation of the extent of parallelism.

A single extent of parallelism can be associated with each simple or structured statement of the language which involves one, or more than one, parallel variable; this must be less than or equal to the declared extent of parallelism for the parallel variables involved. Hence, during program execution, the smallest unit for which the extent of parallelism can be defined is the single assignment statement; this does not exclude the use of scalar and parallel variables in the same statement but facilitates testing, data alignment, etc., of the parallel variables; for example, it avoids the ambiguity involved in the multiplication of two 
parallel variables with different extents of parallelism. Thus the indices, including any alignments, are determined for each statement before execution and if they are found to be incompatible this gives rise to an error.

In those situations where the extent of parallelism will not change for several statements, the within construct can be used to define it. In addition, structured statements such as the if, case, while, and for constructs can define the extent of parallelism for the statements which they are applied to (if they involve parallel variables). These constructs are collectively referred to as extent-setting constructs; in effect, they represent an extension of program-structuring constructs to the lockstep parallel environment.

\subsection{The Within Construct}

In order to avoid repeatedly indicating the extent of parallelism for a series of assignment statements in which the extent will not change, the within construct has been introduced. This, in turn, will avoid a calculation of the extent of parallelism for each of the statements individually. It takes the form

within specifier do statement

where "specifier" is either an index set identifier or an explicit extent of parallelism. The specifier defines the extent of parallelism for the "statement," and the sharp symbol \# is used to indicate the extent of parallelism. For example, consider the following code fragment:

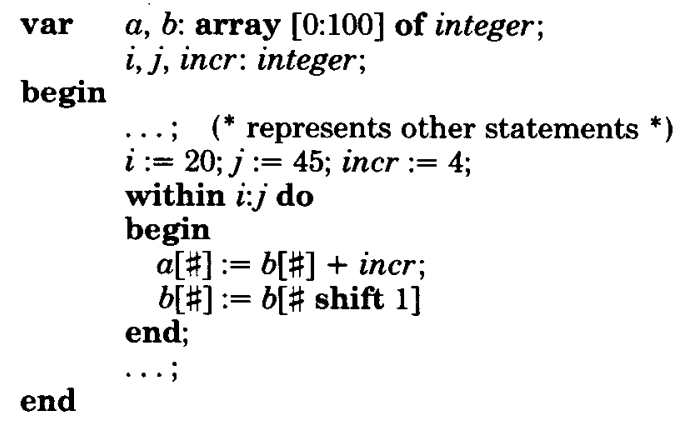

The sharp abbreviation symbol represents the extent of parallelism defined by the specifier, i.e., the ordered integer set $i$ to $j$ inclusive.

This construct will avoid a calculation of the extent of parallelism until another extent-setting construct is encountered or the construct is exited. If another extent-setting construct is encountered, the current extent of parallelism is stacked and the new extent evaluated and applied; when the new construct is exited, the original extent of parallelism is unstacked and it is then applied to the rest of the statements. This is the rule which governs the nesting of all extentsetting constructs.

The within construct also serves another purpose when it is embedded in a loop; the specifier can consist of variables which are changed each time through the loop, thus, for example, enabling the examination of various subgrids within a larger grid.

It is appropriate at this point to digress from the presentation of the language in order to mention briefly the implementation of the extent of parallelism. This 
digression is intended to give the reader an appreciation of the central role of this concept both for the language and for the various parallel architectures.

On the Illiac IV, such a concept can be implemented by setting the "mode" bits; one mode bit is associated with each processing element and the status of the bit determines whether that processing element will execute instructions or not. At present, the user must, in CFD and Glypnir, manipulate these bits directly. On the Star-100, the extent of parallelism can be represented by the control vector which determines which elements of a data structure are to be operated upon. At present, in Star Fortran, the user invokes a subroutine call to set the control vector. On the Cray-1, there is a vector mask register which can be used to exclude a data element from execution; at present in CFT, the user has no means of setting this register.

\subsection{Structured Statements}

To allow for those situations where selection or repetition is concerned, the structured programming concepts of if, case, while, and for were expanded to enable the test or loop variables to contain parallel as weli as scalar variables. In the latter case, the extent of parallelism for any of the constructs' statements involving parallel variables must be explicitly defined or must be inherited from an enclosing extent-setting construct. If parallel variables are involved in the test or loop variables, then their extent of parallelism must be the same. The extent of parallelism for the construct's statements is determined from these parallel variables and the sharp abbreviation symbol used to represent the appropriate extent of parallelism in the statements. Further illustration of these constructs using parallel variables is now considered.

(a) Selective Statements. Selective statements are used to spread the extent of parallelism between two or more execution paths, as determined by a test expression in the if or case constructs.

If a test expression involves parallel variables, the test is evaluated for each indicated element of the variables and if it is found to be true then the appropriate statements of the construct are applied to that element, e.g.,

$$
\text { if } a[0: 49]>b[0: 49] \text { then } a[\#]:=a[\#]-1
$$

In this example, 50 elements of $a$ are tested to see which are greater than the corresponding elements of $b$; those elements that are greater are decremented by 1 .

If the test involves data alignment, a base on which to align must be clearly indicated in the test variables, so that there is no ambiguity in the extent of parallelism of the statements, e.g.,

$$
\begin{array}{r}
\text { if } a[10: 90]<a[10: 90 \text { shift }-1] \text { then } a[\#]:=a[\#]+1 \\
\text { else } a[\#]:=a[\#]-1
\end{array}
$$

Those elements of $a$ in the range 10 to 90 which are less than their left neighbor have their values incremented by 1 and the remaining elements are decremented by 1 .

The connectors and (conjunction), or (disjunction), not (negation) plus the "parallel" quantifiers any and all can be used in a test. The latter two are used to apply a test across an extent of parallelism, i.e., if at least one element obeys 
the test or if all the elements obey the test, respectively. Since these parallel quantifiers reduce to a single value for the test, they are governed by the rule for a scalar test, that is, the extent of parallelism must be explicitly defined or inherited from an enclosing extent-setting construct. For example,

$$
\text { if any }(a[40: 70]>0) \text { then } a[40: 70]:=a[40: 70]-1
$$

if one (or more) of the elements 40 to 70 of $a$ are positive, then all the elements 40 to 70 of $a$ will have one subtracted and

$$
\begin{aligned}
& \text { if all }(a[2: 29]>b[2: 29]) \text { then } b[2: 29]:=0 \\
& \text { else } a[2: 29]:=0
\end{aligned}
$$

if all the elements 2 to 29 of $b$ are less than their corresponding elements in $a$, then the elements 2 to 29 of $b$ are put to zero; otherwise, the elements 2 to 29 of $a$ are put to zero.

The case construct has also been expanded to allow parallel variables in the labeled statements and a parallel variable as the selector variable. In the latter situation each element of the selector variable selects a labeled statement as determined by the value of that element; this causes the extent of parallelism to be distributed among the labeled statements which are then successively executed. For example,

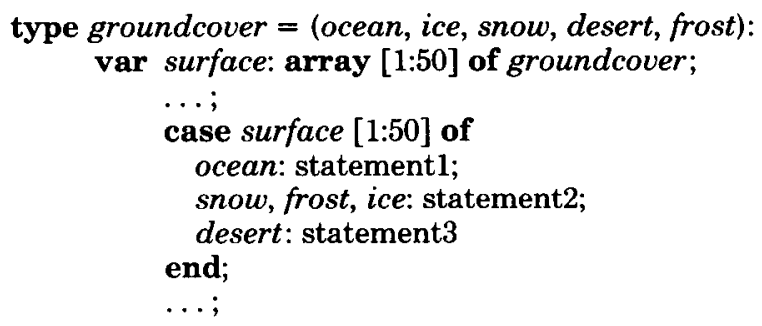

The sharp abbreviation must be used to represent the extent of parallelism for each of the "statements" since they are determined by the current values of the selector variable surface. The possible values of surface are represented by the labels and the current values determine which statements are applied to that element. The extent of parallelism is thus spread among the labeled statements so that each element is associated with one of the statements only.

(b) Repetitive Statements. There are two types of repetitive statements, depending upon whether the number of times the statement is to be executed is known before the statement is encountered or whether the number is dependent on conditions generated by the statement.

(i) The first case is for an unknown number of times. This is achieved by using the while construct. As with the selection constructs, the test can include parallel or scalar variables or both. The extent of parallelism for the test variables must be the same and the result of its evaluation determines the extent of parallelism for the statements, which is represented by the sharp symbol. For example, in the statement

$$
\text { while } a[1: 50]<b[1: 50] \text { do } a[\#]:=a[\#]+1
$$

the sharp symbol identifies those elements of $a$ which are less than their ACM Transactions on Programming Languages and Systems, Vol. 1, No. 2, October 1979. 
corresponding element in $b$ on each occasion that the comparison of elements is performed; only those elements of $a$ have their value increased by 1 . Hence the sharp symbol represents a nonincreasing number of elements as a result of successive executions of the test. Execution terminates when all the elements of $a$ are greater than or equal to their corresponding element in $b$.

The parallel quantifiers any and all can be used with parallel test variables in which case the extent of parallelism must be explicitly defined in the statements of the construct (as with a test which involves scalar variables only). For example, in the situation

$$
\text { while any }(a[1: 50]<b[1: 50]) \text { do } a[1: 50]:=a[1: 50]+1
$$

all the elements of $a$ are incremented by 1 until none of the elements of $a$ are less than their corresponding element in $b$.

(ii) The second type of repetition is for a known number of times and is indicated by the for construct, e.g.,

$$
\text { for control:= start to finish do statement. }
$$

The multiplication of an $m \times n$ matrix $a a$ by a vector $w$ of length $n$ can be formed as follows:

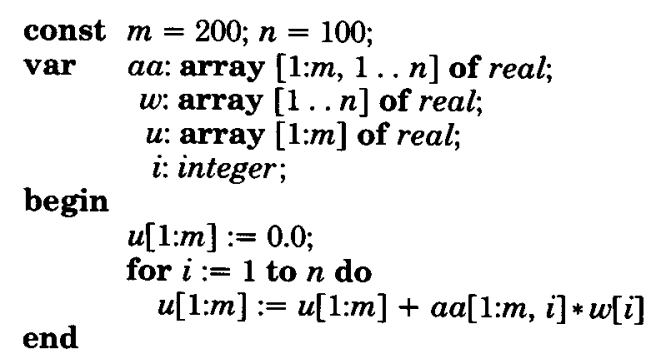

A by clause has been introduced for those situations where a constant increment other than unity is required, e.g.,

for control := start by increment to finish do statement.

The control, start, and finish variables can be either scalar or parallel variables and can be used with either a scalar or parallel constant increment; the extent of parallelism must be the same for all parallel entities. This extent is then represented by the sharp abbreviation in the statements of the for construct. As with independent indexing a one-dimensional parallel integer array is used as the parallel loop index enabling a different fixed number of iterations to be applied to each element of a parallel array. For example,

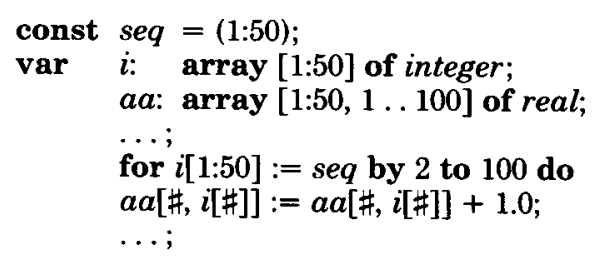

In this example the parallel index $i$ is initialized with the values 1 to 50 . After ACM Transactions on Programming Languages and Systems, Vol. 1, No. 2, October 1979. 
each execution, all the values are increased by 2 until they reach 100 . Hence the extent of parallelism decreases as the number of times the statements are executed increases.

\subsection{Functions and Procedures}

Functions and procedures can be declared using the data declarations and statements previously defined; the maximum extent of parallelism of all variables must be known at compile time. The Pascal scope rules for procedures and functions apply; hence local variables cannot have their extent of parallelism altered by a function or procedure call.

The formal parameter list for both functions and procedures was expanded to allow for parameters which are parallel variables. The actual parameters can then be either of the same extent of parallelism or a section of the same extent of a larger parallel variable. Only procedures and functions involving scalar variables may be parameters.

In the case of a function either a scalar or parallel variable can be returned as a result of its execution; the extent of parallelism can be different from that of the parameter(s). Procedures can be used to return one or more results which can be either scalar or parallel variables or a mixture of both.

In addition, several standard functions have been defined which perform frequently required operations; they can be divided in two classes:

(i) those which can be applied to each element of a parallel variable e.g., the cosine function;

(ii) those which yield a single result using several elements of a parallel variable, e.g., the sum function which adds the elements of the parallel variable which is its argument.

\subsection{Examples}

Two examples are now considered to illustrate further the use of some of the above features.

(i) The first example represents the main loop in a Gauss-Jacobi solution of Poisson's equation and in particular illustrates the use of an index set and an operand alignment operator.

The basis of the method is that each interior point of a rectangular grid $a a$ is updated using its adjacent neighbors at the north, south, east, and west positions. This is achieved in turn for each column in parallel and the process is repeated until successive values for all the points differ by a required tolerance epsilon. The values used to calculate the new values are those obtained as a result of the previous calculation.

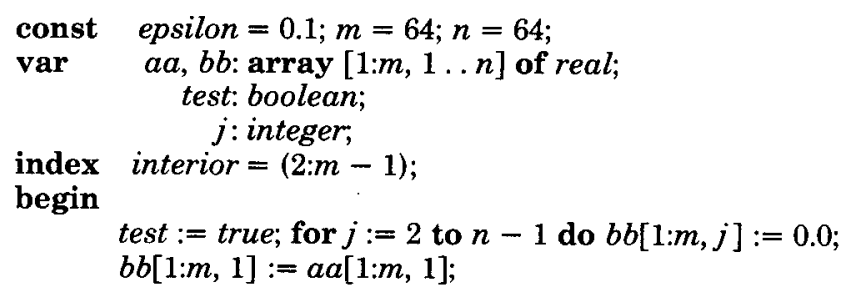




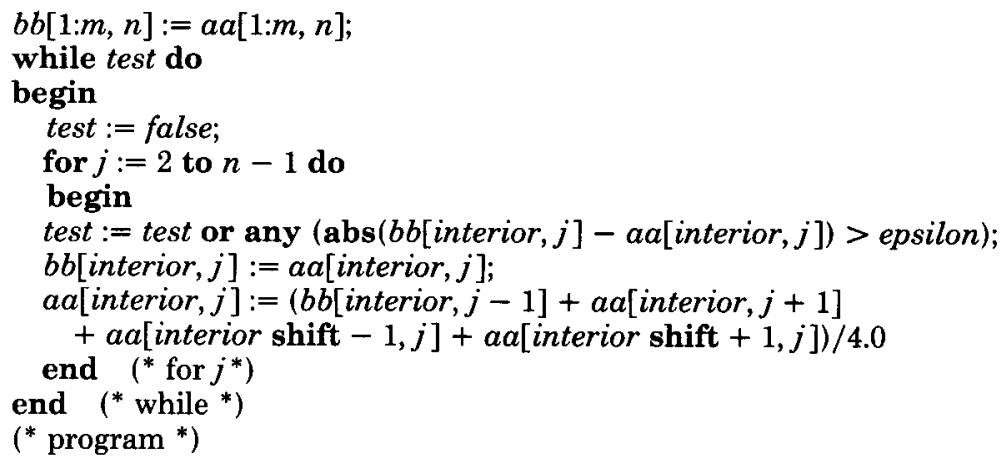

(ii) The second example transposes an $n \times n$ matrix in the fast processing store and illustrates the use of the within construct and independent indexing.

The algorithm works by moving $n$ elements of the array $a a$ to their new positions in the array $b b$ in parallel. The $n$ elements are selected by diagonals and are moved to their reflected positions about the main diagonal. The $n$ old and $n$ new positions are determined by the indexing arrays $i$ and $j$, respectively. Note: An inplace transpose can be performed which requires less storage.

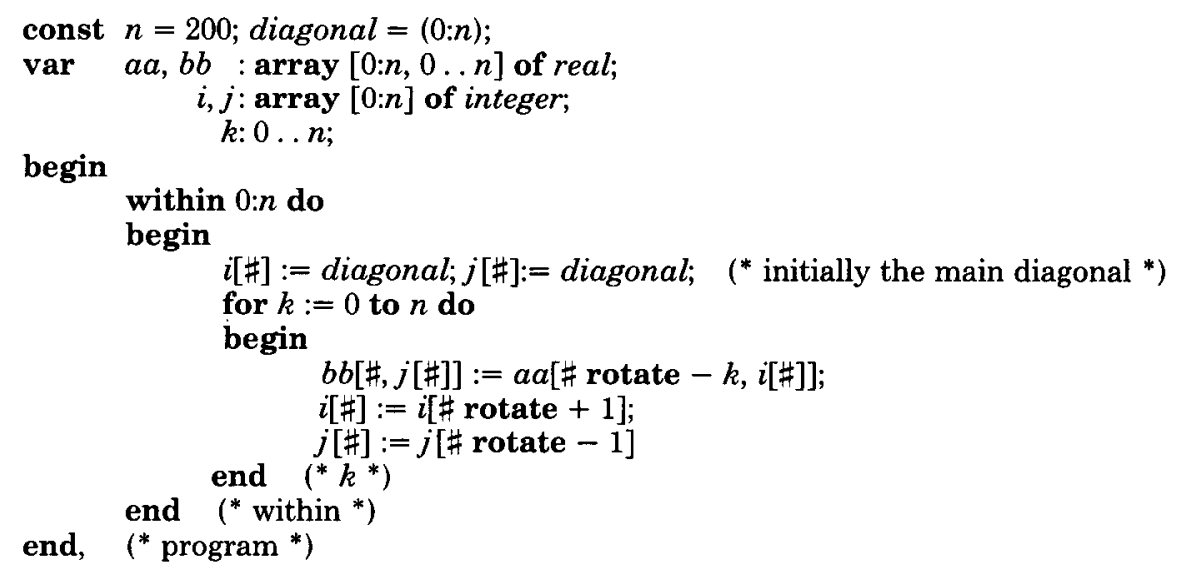

(* the index $i[\#]$ selects two off diagonals in $a a$, an upper and a lower off diagonal; their corresponding positions in the transposed matrix $b b$ are indicated by $j[\#]$. The other indices of $a a$ and $b b$ indicate the row coordinates of these diagonals*)

The examples illustrate how an extent of parallelism is associated with each data declaration and subsequently manipulated by the statements.

\section{AUTOMATIC BUFFERING}

The current parallel computers are often required to handle large databases which cannot be held in the fast store; the backing store is used to hold the database and the programmer has to ensure that the data are brought into the fast store in units which can be easily stored and manipulated (in the Star-100 this process is handled by a virtual memory). In order to avoid the considerable latency delays associated with the backing store, some form of buffering is necessary. 
To automate the management of the memory, it is important to determine either from the user or by the compiler the size of the working set; the working set is the minimum amount of the database required to be resident in the fast store so that processing can continue without excessive interruptions. On the basis of such information, the fast store can be divided into buffers and processing can be overlapped with backing store transfers. Thus the compiler rather than the user is responsible for the organization of data transfers.

The data declaration is used to indicate such information; for example, consider the array

$$
\text { var aaa: array }[1: m, 1 \ldots n, 1 \ldots p] \text { of integer; }
$$

If the product of the dimensions $m \times n \times p$ is such that it is not possible to hold the array aaa in the fast store, then the user should, employing his knowledge of the use of $a a a$, indicate the minimum working set size. This can be achieved by appending a positive integer to the index that changes most slowly in the course of the calculation. For example, the declaration

$$
\text { var aaa: array }[1: m, 1 \ldots n(3), 1 \ldots p] \text { of integer; }
$$

indicates that the user wishes to sweep through the database in the third index direction and requires three columns of the second index when making an update; the minimum working set size is $m \times 3 \times p$ (in the case of the Star-100 this information can be used to determine the page transfer size or to pack data on a page). Hence a small change in the syntax will guide the automatic allocation of temporary storage in the fast store. For example, single, double, or multiple buffering can be used to divide up the fast store to obtain an overlap of processing and data fetching based on the working set size for the database.

A program can be tested using a smaller database and a small syntax change in the data declaration is all that is required to move to a larger database. For example, in the Gauss-Jacobi solution of the last section, if the size of the database is too large, only the declaration of $a a$ and $b b$ should be changed to help with the movement of the data, e.g.,

$$
a a, b b: \operatorname{array}[1: m, 1 \ldots n(5)] \text { of real; }
$$

\section{IMPLEMENTATION}

The features of Actus have been described using a syntax similar to that of Pascal; this was due, in part, to a plan to use an existing Pascal compiler for its implementation.

Since it has been possible to express the constructs of Actus in sequential Pascal, it is therefore possible to build a preprocessor for Actus programs. This will enable the debugging of Actus programs at an installation which does not have a parallel machine of this type but does have a Pascal compiler. After debugging, such programs could be sent to an installation which does have a parallel machine of this type, by means of the ARPA network, for example.

In this way a wider range of potential users can be given the opportunity of being involved with existing parallel machines. This could also promote the exportability of codes developed on one parallel processor to another. Such a preprocessor is currently being constructed in Pascal. 
A frequently used procedure for the implementation of a high level language for current parallel computers is to use an auxiliary computer to perform the compilation of the programs. A similar approach has been adopted for the implementation of Actus on the Illiac IV.

More specifically, a Pascal P-compiler is being used in the creation of the Actus compiler. This $\mathrm{P}$-compiler is being modified and enhanced with the new features to form an Actus P-compiler which also generates code for a hypothetical stack computer. Since this code is machine independent, the Actus P-compiler can be used as a basis for the implementation of Actus on other parallel machines.

Using a Pascal compiler as the host compiler should significantly reduce the implementation time. Also, many of the implementation decisions, e.g., the parameter passing mechanism, are already decided and proven in practice, and compatible with the extensions. Finally, all the compile time and run time checking facilities of Pascal are inherited and they can be expanded to check the new language features also.

Preliminary results, of the implementation indicate that the features of Actus can be mapped onto the instruction set of the Illiac IV. The extent-setting patterns that will arise due to the control structures can be implemented by means of the mode bits, where the status of each of the 64 mode bits determines whether its corresponding processing element is active or not. This, in turn, determines whether a particular element of a parallel array is to be acted upon or not. The Illiac IV has sufficient registers to handle the nesting of a number of extent-setting constructs.

The layout of the data in the main store to face the processing power, i.e., the processing elements, requires that some base point be chosen so that parallel variables have the same indices aligned opposite the same processing elements. For example, if processing element zero is chosen as index base point 1 , then all indices which are an incremental distance of 64 (either positive or negative) should be placed in the store of processing element zero, and similarly for all the other processing elements ( 1 to 63 ).

Thus an array with a parallel index range greater than 64 will be stored in consecutive rows with all multiples of 64 in the same processing element's store. The processing of such arrays will require repeated application of the processing elements to the number of rows involved, with allowance being made for the end conditions.

For example, in the assignment statement $a[1: n]:=0$ where $n>64$, this will require ( $n$ div 64) applications of the 64 processing elements to the consecutive rows plus ( $n \bmod 64)$ processing elements active in another application of the processing elements ( $n$ not a multiple of 64). A nonuniform extent of parallelism $a[\#]:=0$ will require the status of the processing elements to be enabled and disabled accordingly.

Intercommunication between parallel arrays using the alignment operators requires that extra storage is set aside whenever the index range is greater than 64; this temporary storage is governed by the distance the data is to be moved. It is required to ensure that the updating of the end of one row with the beginning of another (or vice versa) is done properly.

The control unit of the Illiac IV, which has no floating point arithmetic 
capability, can be used to manipulate the data stack in the course of program execution.

These are the features of Actus which are currently being implemented in the first phase of the compiler for the Illiac IV at the Institute for Advanced Computation.

\section{SUMMARY}

The objective of the research reported here was to design a high level language which is appropriate for both vector and array parallel computers, and at the same time to reflect many of the advances which have been made in the design and implementation of high level languages in general. The language is therefore unsuitable for other types of parallel processors, such as multiprocessor configurations.

The first objective was achieved by introducing the concept of the extent of parallelism, whose maximum size is defined in the data declarations and subsequently manipulated (in parallel) either in part or in total by the statements and constructs of the language. Using this concept, it was found to be possible to adopt a unified approach for both types of computers. The second objective was achieved by modifying existing data and program structuring constructs of Pascal to accommodate the special demands of a parallel environment.

The advantage of the language is that a problem's parallel nature is expressed directly in the syntax of the language which, in turn, makes efficient use of the machine's computational resources. The user, therefore, does not have to get involved with the hardware of these machines or the compiler dependencies of the languages; this involvement has been necessary when using current or previous languages for such machines. Thus the language brings the benefits of program and data structuring to the large scale scientific computation area and still maintains the advantages of compile time and run time checking.

\section{ACKNOWLEDGMENTS}

The author wishes to thank the staff of the Institute for Advanced Computation for the help he received in the course of this work. In particular, D. Stevenson's continual penetrating questions and knowledge of the architecture of these parallel computers were of invaluable assistance. The author would also like to thank the referees for their careful reading of the manuscript and for a number of suggested improvements.

\section{REFERENCES}

1. Barnes, G.H., Brown, R.M., Kato, M., Kuck, D.J., Slotnick, D.L., and Stokes, R.Q. The ILLIAC IV computer. IEEE Trans. Comptr. C-17 (Aug. 1968), 746-757.

2. Feierbach, G.F., and Stevenson, D.K. The Phoenix array processor. Proc. 17th Annu. Tech. Symp., June 1978, pp. 3-10.

3. Holland, S.A., and Purcell, C.J. The CDC Star-100: a large scale network oriented computer system. Proc. 1971 IEEE Conf., Sept. 1971, pp. 55-65.

4. Jensen, K., And Wirth, N. Pascal: user manual and report. Lecture Notes in Computer Science, Vol. 18. Springer-Verlag, Berlin, 1974.

5. KIskI, T. Tensor/ILLIAC IV project. Rep. UCRL-51467, Lawrence Livermore Lab., U. of California, 1973. 
6. KNIGHT, J. The SL/1 programming manual. NASA Langley Res. Ctr., March 1978.

7. LAWRie, D.H., LAYMAN, T., BAER, D., ANd RANDAL, J.M. Glypnir-a programming language for ILLIAC IV. Comm. ACM 18, 3 (March 1975), 157-164.

8. Millstein, R.E. Control structure in ILliaC IV FORTRAN. Comm. ACM 16, 10 (Oct. 1973), 622-627.

9. Nori, K.V., Ammann, U., Jensen, K., and Nägeli, H. The Pascal (P) compiler: implementation notes. Institut für Informatik, Eidgenössische Technische Hochschule, Zürich, 1975.

10. Russell, R.M. The Cray-1 computer system. Comm. ACM 21, 1 (Jan. 1978), 63-72.

11. Star programming manual. Control Data Corp., 1976.

12. Stevens, K. CFD-a FORTRAN-like language for the ILLIAC IV. Sigplan Notices (March 1975), 72-80.

13. Stevenson, D.K. Programming the ILLIAC IV. Tech. Rep., Dep. Comptr. Sci., Carnegie-Mellon U., 1975.

14. Wirth, N. The programming language PASCAL. Acta Informatica 1 (1971), 35-63.

15. Wulf, W.A., AND Bell, C.G. C.mmp-a multi-mini processor. Proc. AFIPS 1972 Fall Joint Computer Conf., 1972, pp. 765-777.

Received August 1978; revised February 1979 